\title{
Call for collaboration in dementia research
}

$\mathrm{B}$ usiness as usual is not an option in the global fight against dementia, urged experts at a legacy event held in Ottawa this fall. They called for collaboration and regulatory flexibility as well as financial incentives for researchers and drugs companies to sustain long-term investments toward finding a cure.

Traditional research and development methods haven't delivered results, and may not show progress before health systems are overwhelmed, World Dementia Envoy Dennis Gillings told some 200 delegates to the Canada-France Global Dementia Legacy Event. "There are 44 million people with dementia around the world and yet just three drugs have been developed in over 15 years."

Part of the problem is how much is still unknown about the disease. "Dementia is probably tens of diseases or more, and the solution may be a combination or multiple therapies," said Gillings. "That means the research effort is very substantial and some therapies won't show their full merits until the synergies with others have been discovered."

In particular, "we need more knowledge about targets in the brain that the pharmaceutical industry can build drugs to stimulate." But identifying those targets comes under the rubric of basic neuroscience, and attrition is high in discovery and early development.

Mounting evidence also suggests that changes in the brains of people with Alzheimer disease can begin decades before symptoms appear. One explanation for recent phase- 3 trial failures is that patients took the drugs too late in the disease process, when they had already suffered substantial brain cell death. In 2013, trials began studying patients in earlier stages.

More broadly, however, "there was a sense that industry was starting to walk away from this question," Federal Health Minister Rona Ambrose told reporters at the Sept. 11-12 event.

Panelists agreed that collaboration was a first step to lowering the investment risks associated with dementia research. That starts with companies

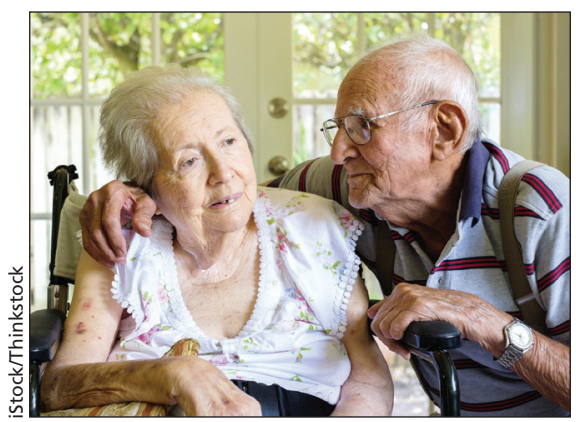

Dementia is devastating for patients and their caregivers, but the high risks of traditional research and development methods are stalling progress to a cure.

pooling data to reduce inefficiencies and learn from each other's mistakes, said Michel Goldman, executive director of the European Union's Innovative Medicines Initiative.

For example, "five companies developing new antipsychotics that pooled their data ... demonstrated you can reduce by $40 \%$ the number of patients you have to enrol in a trial to demonstrate drug efficacy."

Others suggested collaboration to standardize contracts and regulatory requirements between companies, academia and governments, and across international borders.

Still others argued that dementia drugs may merit a special regulatory category with expedited review, as in certain cases with medicines for HIV, oncology and orphan diseases.

This suggestion drew ire, however, from health regulators in the room. "In this era of teamwork, we don't want the umpire to be an advocate," said Robert Cushman, director general of the Biologics \& Genetic Therapies Directorate at Health Canada.

However, Cushman agreed that international collaboration to review drugs might ease the burden on smaller regulators. "We have to survey the same waterfront, the same number of medicines and the same complications that the US Food and Drug Agency does with $10 \%$ of their budget and size." Lauren Vogel, CMAJ

CMAJ 2014. DOI:10.1503/cmaj.109-4903 